\title{
Maladies émergentes transmises par les tiques
}

\author{
Ingomar Mutz
}

Abteilung für Kinder und Jugendliche, Landeskrankenhaus Leoben, Leoben, Autriche

\author{
Mots-clés \\ Anaplasmose $\cdot$ Babésiose $\cdot$ Borréliose $\cdot$ Flavivirus $\cdot$ Maladies \\ causées par les rickettsies · Fièvre pourprée · Encéphalite \\ transmise par les tiques - Maladies transmises par les \\ tiques - Tularémie
}

\section{Résumé}

Les infections transmises par les tiques surviennent dans le monde entier et sont bien connues depuis plus d'un siècle. Certaines d'entre elles sont très courantes alors que d'autres sont extrêmement rares. Les techniques modernes de génétique moléculaire (dont une plus grande disponibilité de la réaction en chaîne par polymérase $(\mathrm{PCR})$ (de l'anglais polymerase chain reaction) ont permis la découverte et la classification de nouveaux pathogènes, notamment de l'ordre des rickettsies. Le présent article s'intéresse aux agents viraux, bactériens et protozoaires responsables de maladies chez I'homme. La plupart des morsures de tiques et donc des maladies qui en résultent, surviennent durant la période la plus chaude de l'année. Les réservoirs naturels de ces maladies présentent une grande variation géographique. L'origine d'une morsure de tique n'est pas fiable car elle passe souvent inaperçue. Les maladies transmises par les tiques les plus courantes sont la borréliose de Lyme et l'encéphalite due à un flavivirus. Les infections virales se manifestent le plus souvent par les signes neurologiques de l'encéphalite tandis que les infections causées par les rickettsies se caractérisent par des poussées de fièvre (biphasique), des symptômes systémiques liés à une infection et une éruption cutanée avec présence de pétéchies. Certaines maladies transmises par les tiques peuvent être prévenues par la vaccination, telle que l'encéphalite. Dès les premiers signes cliniques, les infections non virales devraient être traitées très tôt par des antibiotiques afin d'obtenir les meilleurs résultats. Les maladies transmises par les tiques peuvent être fatales. Les taux de mortalité sont variables; ils dépendent de la virulence du pathogène, de l'absence de recours aux mesures préventives ou d'un retard dans le diagnostique et le traitement.

Copyright $\odot 2010$ Nestec Ltd., Vevey/S. Karger AG, Basel

\section{Introduction}

Les infections transmises par les tiques sont bien connues depuis plus d'une centaine d'années. Leur incidence est variable selon la période de l'année et la situation géographique, et dépend de circonstances variées telles que les réservoirs animaux, le climat, les conditions écologiques et le mode de vie. Les mesures préventives comme l'utilisation de répulsifs et le port de vêtements couvrant la plupart des parties du corps n'offrent qu'une protection limitée. La prévention par le vaccin n'existe que pour certaines infections et n'est pas toujours suffisamment utilisée.

Depuis de nombreuses années, des progrès constants ont été réalisés et ont permis une clarification de l'étiologie et du traitement des pathogènes transmis par les tiques; grace aux techniques modernes de génétique moléculaire, on continue à découvrir et à classer de nou-

\section{KARGER}

Fax +4161306 1234

E-Mail karger@karger.ch

www.karger.com (c) 2010 Nestec Ltd., Vevey/S. Karger AG, Basel

0250-9644/09/0673-0123\$26.00/0

Accessible en ligne à: www.karger.com/anf
Prof. Dr. Ingomar Mutz

Schaldorferstrasse 2

AT-8641 St. Marein i.M. (Austria)

E-Mail mutz.ingomar@speed.at 
Tableau 1. Maladies humaines véhiculées par les tiques [2]

\begin{tabular}{ll}
\hline Maladie & Agent \\
\hline Morsure de tique & \\
Paralysie par piqûre de tique & Holocyclotoxin \\
\hline Fièvre à tiques du Colorado & Coltivirus \\
Fièvre hémorragique de Crimée-Congo & Nairovirus \\
MFK & Flavivirus \\
Fièvre hémorragique d'Omsk & Flavivirus \\
Encéphalomyélite de Powassan & Virus de Powassan, virus de la tique du cerf \\
ETT & Flavivirus \\
\hline Typhus de Sibérie & R. sibirica \\
Fièvre boutonneuse japonaise ou orientale & R. japonica \\
Fièvre boutonneuse méditerranéenne & R. conorii \\
Typhus du Queensland & R. australis \\
FPMR & R. rickettsii \\
Fièvre pourprée du groupe des rickettsies & R. helvetica, $R$. monacensis \\
Lymphadénopathie transmise par les tiques & R. slovaca \\
\hline Anaplasmose granulocytaire humaine & A. phagocytophilum \\
EMH & E. chaffeensis, E. ewingii, E. sennetsu \\
\hline Maladie de Lyme & B. burgdorferi \\
Fièvre récurrente & B. duttoni \\
\hline Fièvre Q & C. burnetii \\
Tularémie, fièvre du lapin & F. tularensis \\
\hline Babésiose & B. microti, B. divergens \\
\hline & \\
\hline
\end{tabular}

veaux agents pathogènes. Certaines maladies transmises par les tiques sont très courantes alors que d'autres sont extrêmement rares si bien que seuls quelques médecins y seront confrontés au cours de leur vie professionnelle. Les tests de laboratoire permettant un diagnostic fiable ne sont pas souvent disponibles, sauf dans des laboratoires spécialisés, et prescrire le bon test au bon moment nécessite d'avoir déjà identifié la maladie. Le diagnostic est donc diffficile, il risque de retarder le traitement, et d'avoir un impact négatif sur le résultat final. Pour les médecins s'occupant de ces patients, la formation continue et la consultation en temps opportun de spécialistes s'avèrent importantes.

Les tiques appartiennent à la classe des Arachnides de l'embranchement des arthropodes [1]. Argasidae est le nom donné à la famille des tiques «molles», Ixodidae celui donné à la famille des tiques «dures». Les vecteurs importants dans la transmission de pathogènes humains sont les genres Ixodes, Dermacentor, Amblyomma, Haemaphysalis, Hyalomma et Rhipicephalus de la famille des Ixodidae et le genre Ornithodorus de la famille des Argasidae. La plupart des infections transmises par les tiques surviennent entre mars et octobre. Pendant qu'elles se nourrissent, les tiques peuvent demeurer fixées à leur hôte pendant des heures voire des jours. Une maladie peut être causée par un ou plusieurs agents pathogènes transmis par plusieurs tiques en même temps, l'exposition multiple aux tiques pouvant être liée au mode de vie, et/ou les tiques peuvent transmettre simultanément plus d'un agent infectieux.

Le tableau clinique des maladies transmises par les tiques se caractérise (après une période d'incubation de moins de 10 jours) par une poussée de fièvre soudaine et des symptômes non spécifiques d'une infection systémique tels que frissons, sueurs, céphalées, myalgies, arthralgies, anorexie, nausées et vomissements. La fièvre est souvent biphasique. Les infections virales se manifestent surtout par des signes neurologiques d'encéphalite. Les patients souffrant de fièvre hémorragique de Crimée-Congo, de fièvre hémorragique d'Omsk, ou de maladie de la forêt de Kyasanur (MFK) peuvent développer des hémorragies gastro-intestinales. Les fièvres pourprées causées par les rickettsies se caractérisent par une fièvre souvent biphasique, des symptômes systémiques 
d'une infection et fréquemment mais pas toujours par une éruption maculopapulaire diffuse avec présence de pétéchies et purpura. Les infections par les rickettsies peuvent également présenter un ulcère cutané local ou une escarre (tache noire) au niveau de la morsure de la tique. La borréliose de Lyme est un véritable «caméléon symptomatique» avec des signes et des symptômes variés, qui peuvent donner lieu à un diagnostic clinique trompeur. Elle est accusée, à tort, d'être à l'origine de nombreuses plaintes non associées. Une pneumonie est très courante dans les cas de fièvre Q et de tularémie. La tularémie se présente comme une forme systémique ou ulcéroglandulaire de la maladie. La Babésiose se caractérise par une fièvre prolongée et une anémie (tableau 1) [2].

\section{Réactions locales}

\section{Morsures de tiques}

Les morsures de tiques qui sont en fait des piqûres réalisées par le rostre constitué notamment d'un hypostome denté, passent fréquemment inaperçues parce que la salive de la tique contient des substances aux propriétés anesthésiantes et antihistaminiques. Dans la plupart des cas, la piqûre n'entraîne pas de réponse inflammatoire locale. Les morsures de tiques peuvent cependant causer des réactions locales comme une petite papule, un ulcère ou une escarre. Il apparaît rarement des réactions systémiques ou des signes locaux qui peuvent persister, pour se transformer en ce que l'on appelle un «granulome par morsure de tiques». Dans tous les cas la tique devra être enlevée le plus vite possible, sans l'écraser car cela pourrait provoquer l'injection d'agents infectieux dans la peau. Lorsque la morsure de la tique est passée inapperçue, la durée de la morsure n'aura pas systématiquement de conséquences. En revanche, si la morsure de la tique se prolonge au-delà de $24 \mathrm{~h}$, les risques de transmission de Rickettsia conorii, Borrelia burgdorferi ou Babesia sont réels.

\section{Paralysie par piqûre de tique}

La paralysie par piqûre de tique est une paralysie flasque ascendante apparaissant dans les 2 à 7 jours suivant la fixation de la tique. Elle peut être causée par plus de 40 espèces de tiques à travers le monde entier et entraîner la mort de certains animaux, en particulier les vaches et les moutons. Les 5 espèces de tiques d'Amérique du Nord connues comme pouvant être responsables d'une paralysie sont réparties à travers tous les États-Unis. Les cas humains sont rares et généralement observés chez les enfants de moins de 10 ans.

Maladies émergentes transmises par les tiques
La paralysie par piqûre de tique apparaît lorsqu'une tique femelle engorgée et gravide (porteuse d'œufs) produit une neurotoxine dans ses glandes salivaires et la transmet à son hôte lorsqu'elle se nourrit [3]. La plus grande quantité de toxine est produite entre le cinquième et le septième jour de fixation de la tique et ne peut continuer à être libérée qu'en présence de la tique. Une fois la tique retirée, les symptômes diminuent en général rapidement. Pourtant, dans certains cas, une paralysie profonde risque de se développer, pouvant causer la mort avant même que l'on ne se rend compte de la présence de la tique.

Les premiers symptômes se manifestent par une très grande fatigue, un engourdissement des jambes, des difficultés à marcher ou à se tenir debout et des douleurs musculaires. La paralysie se développe rapidement des membres inférieurs vers les membres supérieurs et, si la tique n'est pas retirée, cette paralysie peut par la suite toucher la langue et le visage. Les complications les plus graves se manifestent par des convulsions, une insuffisance respiratoire et, dans $12 \%$ des cas non traités, cette paralysie est fatale.

Le diagnostic est basé sur les symptômes et l'amélioration rapide de l'état du patient une fois que la tique engorgée est retirée. Le traitement consiste simplement à retirer la ou les tiques en train de se nourrir. Il est important de retirer l'ensemble du rostre car il contient les glandes salivaires qui risquent de continuer à infecter le patient, même après avoir retiré le corps de la tique.

\section{Les maladies virales transmises par les tiques}

\section{La fièvre hémorragique de Crimée-Congo}

La fièvre hémorragique de Crimée-Congo est causée par un membre du genre Nairovirus de la famille des $\mathrm{Bu}$ nyaviridae. Le virus est présent en Afrique, en Asie, et en Europe de l'Est chez les bovins, les ovins et chez des petits animaux comme le lièvre. Il a été isolé au Congo en 1956 et dans la région criméenne en 1967 [4]. L’infection chez l'homme est transmise par morsure de tique (le plus souvent du genre Hyalomma) ou par contact avec le sang ou d'autres tissus provenant du bétail contaminé. Le foie est le site préférentiel de réplication du virus et les macrophages et les cellules endothéliales sont aussi des cellules cibles de cette infection virale [5]. La maladie se caractérise souvent par un état fébrile avec céphalées, myalgie et pétéchies (du $3^{\text {ème }}$ au $6^{\text {ème }}$ jour de la maladie), fréquemment suivi d'un état hémorragique avec hépatite nécrosante. Le taux de mortalité est d'environ 30\% [6]. Le dia-

Ann Nestlé [Fr] 2009;67:123-134 
gnostic est réalisé par l'isolation du virus ou par un test ELISA. Aucune mesure préventive (vaccination) ou traitement spécifique n'est actuellement disponible.

\section{La fièvre à tiques du Colorado}

La fièvre à tiques du Colorado se transmet par le Dermacentor andersoni, également responsable de la transmission de la fièvre pourprée des montagnes Rocheuses (FPMR). La période d'incubation est de 0 à 14 jours. Les symptômes sont les suivants: fièvre, frissons, malaises, céphalées, douleur rétro-orbitale, douleur lombaire, myalgie, hyperesthésie, parfois lymphadénopathie et/ou hépatosplénomégalie, plus rarement éruption cutanée, leucopénie (neutropénie plus importante après 5-6 jours). Le diagnostic est réalisé par l'isolation du virus ou par un test ELISA. Aucune mesure préventive (vaccination) ou traitement spécifique n'est actuellement disponible.

\section{Le virus transmis par les tiques du cerf}

Le virus transmis par les tiques du cerf est apparenté au virus de Powassan transmis par les tiques (virus de Powassan lignée II) et est un membre du groupe des flavivirus; il fut d'abord isolé en 1997 en Amérique du Nord chez les tiques Ixodes scapularis [7]. Le virus de Powassan peut causer une encéphalite sévère avec un taux de mortalité pouvant atteindre $60 \%$ et entraîner des séquelles chez les survivants. Le diagnostic est réalisé par l'isolation du virus ou par un test ELISA. Aucune mesure préventive (vaccination) ou traitement spécifique n'est actuellement disponible.

\section{La maladie de la forêt de Kyasanur}

La maladie de la forêt de Kyasanur (MFK) est endémique en Asie du Sud. Elle a été observée pour la première fois dans la forêt de Kyasanur, à Karnataka en Inde. Cette maladie a aussi été décrite chez des singes en 1957, et est donc également connue sous le nom de maladie du singe. La MFK est confinée à la région de Karnataka en Inde où plusieurs centaines de cas se déclarent chaque année. Cependant, un virus similaire au virus de la MFK a récemment été découvert en Arabie Saoudite [8].

Les principaux hôtes du virus à MFK sont des petits rongeurs (musaraignes, rats) mais on peut également les trouver chez les porcs-épics, les chauves-souris et les singes. Le vecteur servant à la transmission de la maladie est le Haemaphysalis spinigera, une tique des forêts.

Après une période d'incubaton de 3 à 8 jours, les patients infectés développent brutalement de la fièvre, souffrent de céphalées, de douleurs musculaires importantes, de douleurs dorsales, ils toussent, sont déshydratés, et présentent des symptômes gastro-intestinaux, une conjonctivite, et des problèmes hémorragiques. Les patients peuvent être victimes d'hypotension artérielle, avec des taux de plaquettes, de globules blancs et de globules rouges anormalement bas. Après une à deux semaines, les symptômes diminuent et certains patients se rétablissent sans complication. Néanmoins, chez la plupart des patients, la maladie est biphasique et une seconde vague de symptômes apparaît au début de la troisième semaine: fièvre et signes d'encéphalite [9]. Le taux de mortalité est de 3 à $5 \%$. La période de convalescence peut durer plusieurs mois, avec des myalgies et un état de faiblesse. Le diagnostic est réalisé par l'isolation du virus ou par un test ELISA. Aucune mesure préventive (vaccination) ou traitement spécifique n'est actuellement disponible.

\section{La fièvre hémorragique d'Omsk}

La fièvre hémorragique d'Omsk est causée par un flavivirus, le virus de la fièvre hémorragique d'Omsk. Ce virus fut détecté en 1945 et, comme pour les autres flavivirus, son génome a été complètement séquencé et analysé [10]. On trouve ce virus en Sibérie Occidentale, principalement parmi les trappeurs de rats musqués. L'infection est transmise par les tiques (Dendrobates reticulatus, Dermacentor marginatus, Ixodes persulcatus) ou directement par les rats musqués ou l'eau contaminée.

Après une période d'incubation de 3 à 7 jours une première poussée de fièvre avec frissons, céphalées et douleurs aux extrémités peut s'accompagner de lésions papulovésiculaires sur le palais souple, de lymphadénopathie cervicale, d'épanchement conjonctival, de signes de pneumonie et d'hémorragie gastro-intestinale; durant la seconde poussée de fièvre, les signes d'une légère encéphalite apparaissent. Le taux de mortalité est inférieur à $5 \%$. Aucune mesure préventive (vaccination) ou traitement spécifique n'est actuellement disponible.

\section{L'encéphalite transmise par les tiques}

L'encéphalite transmise par les tiques (ETT), également connue sous le nom d'encéphalite d'Europe Centrale, est causée par un virus à $\mathrm{ARN}$ appartenant à la famille des Flaviviridae, un sous-groupe du groupe des togavirus. L'infection par ce virus entraîne la production d'anticorps aux propriétés neutralisantes contre la glycoprotéine E de surface.

Le vecteur de ce virus est la tique Ixodes ricinus (ou I. persulcatus en Extrême-Orient), qui transmet le virus par sa salive lorsqu'elle suce le sang. Les réservoirs naturels sont des petits rongeurs et le gibier. Dans les régions 
endémiques, 0,2 à $0,5 \%$ des tiques sont porteuses du virus. Contrairement à ce qui se passe avec la transmission de B. burgdorferi, il suffit que la tique suce le sang pendant un laps de temps très court pour permettre la transmission du virus à l'homme. Plus rarement, le virus peut être transmis par le lait de chèvre non pasteurisé et contaminé.

L'ETT fut rapportée pour la première fois en Europe en 1931 lorsque Schneider [11] (en Autriche) décrivit une méningite séreuse d'étiologie inconnue. Dans la région la plus orientale de l'Union Soviétique la maladie fut décrite en 1934. Le virus fut isolé et la transmission par les tiques démontrée en 1937.

Plus de 10000 cas d'ETT surviennent chaque année en Europe. Les pays les plus infectés sont l'Autriche, la Croatie, la République Tchèque, la Hongrie, la Slovénie, et la Slovaquie. La maladie concerne également les régions au sud de l'Allemagne, la Finlande, la Pologne, la Suède, et la Suisse. À présent, les pays du Benelux et le Portugal sont les seuls pays européens où aucun cas d'ETT a été décrit. Un très grand nombre de cas se déclarent également dans la partie asiatique de la Russie, dans la partie septentrionale de la Chine et dans les régions avoisinantes.

La répartition saisonnière de l'ETT dépend de l'activité des tiques dans la nature, en fonction des conditions climatiques telles que la température et l'humidité. En Europe Centrale, l'activité maximale est observée entre mai et octobre. Le nombre de cas varie d'une année à l'autre en fonction du climat et des activités de loisir. En raison des changements climatiques, la maladie semble s'étendre vers les régions occidentales et montagneuses. Au dessus de $1000 \mathrm{~m}$ d'altitude où la température annuelle moyenne est inférieure à $8^{\circ} \mathrm{C}$, les tiques et le virus n'existaient pas jusqu'à récemment lorsque une infection par l'ETT fut rapportée à une altitude de $1600 \mathrm{~m}$.

Il peut s'agir d'une maladie modérée ou asymptomatique ou, dans $10 \%$ des cas, elle peut se manifester sous la forme d'une méningite, d'encéphalite, de myélite ou d'une combinaison de plusieurs de ces symptômes. La maladie est souvent plus modérée chez les enfants, bien que des cas d'encéphalite accompagnée de séquelles neurologiques permanentes aient été décrits [12]. Aucun traitement antiviral n'est disponible.

Pour le diagnostic, un test ELISA est disponible. Il a une réaction croisée avec le virus de l'encéphalite japonaise $B$ mais l'utilisation d'un test de neutralisation permet de contourner ce problème.

L'ETT est une maladie que l'on peut prévenir par la vaccination [13]. Le vaccin couvre les trois sous-types de la maladie (sous-types européens, de Sibérie Centrale, et
Tableau 2. Rickettsiales

\begin{tabular}{llll}
\hline Ordre & Famille & Genre & Espèces \\
\hline $\begin{array}{l}\text { Rickett- } \\
\text { siales }\end{array}$ & $\begin{array}{l}\text { Rickettsi- } \\
\text { aceae }\end{array}$ & $\begin{array}{l}\text { Rickettsia } \\
\text { Orientia }\end{array}$ & $\begin{array}{l}\text { R. acari et autres } \\
\text { O. tsutsugamushi }\end{array}$ \\
\cline { 2 - 4 } & $\begin{array}{l}\text { Anaplas- } \\
\text { mataceae }\end{array}$ & $\begin{array}{l}\text { Anaplasma } \\
\text { Ehrlichia }\end{array}$ & $\begin{array}{l}\text { A. phagocytophilum et autres } \\
\text { E. chaffeensis et autres }\end{array}$ \\
\hline
\end{tabular}

d'Extrême-Orient). De 1970 à 2008 un total de 8848 cas d'ETT furent diagnostiqués en Autriche. La grande majorité des patients dont le diagnostic avait été confirmé par des tests sérologiques furent hospitalisés. Malgré des taux de couverture et d'efficacité vaccinales très élevés (respectivement 88 et $98 \%$ ), 50 à 100 cas sont enregistrés chaque année en Autriche [14].

\section{Maladies causées par les rickettsies et transmises par les tiques}

Auparavant, toutes les bactéries intracellulaires étaient appelées «rickettsia» $[15,16]$. Suite à l'analyse de la sousunité $16 \mathrm{~S}$ de leur ARN, cette appellation systématique dut être modifiée et l'ordre des Rickettsiales fut divisé en deux familles (tableau 2) [17].

Après leur inoculation, les rickettsies se multiplient dans les cellules endothéliales des petits vaisseaux sanguins et se disséminent largement dans le système vasculaire. Les symptômes des infections causées par les différentes rickettsies sont similaires. Après une période d'incubation de 6 à 7 jours, le patient présente de fortes fièvres pouvant durer 2 à 3 semaines, une éruption maculopapulaire au niveau des extrémités et, dans la plupart des cas, une thrombocytopénie. La lésion causée par la morsure peut parfois devenir nécrotique. Pour détecter une rickettsiose, il est essentiel de pratiquer un examen clinique minutieux et de réaliser une investigation épidémiologique. La présence d'une éruption cutanée caractéristique est souvent le meilleur indice pour établir le diagnostic. Un simple test diagnostique sérologique peut prendre plus d'une semaine et donc, la recherche de la présence des bactéries sur une biopsie de peau prélevée sur une lésion, par un test de détection d'ADN ou en utilisant des anticorps fluorescents ou marqués à la péroxidase, doit être réalisée pour un diagnostic plus rapide. Les rickettsies sont sensibles à la tetracycline; la doxycycline est par conséquent le médicament de choix et le traitement de- 
vrait être commencé le plus tôt possible, avant le cinquième jour de la maladie, pour obtenir un résultat optimal et réduire la mortalité. Lorsque la tetracycline est contre-indiquée (jeunes enfants, femmes enceintes), il est possible d'utiliser des antibiotiques tels que le chloramphénicol, la josamycine, ou une association de rifampicine et d'érythromycine. Avant l'existence d'un traitement antibiotique, la mortalité due à la FPMR était de $25 \%$. Cette mortalité peut toucher encore de 5 à $7 \%$ des cas si le traitement est débuté tardivement. Les autres maladies dues aux rickettsies (autres que la FPMR) sont souvent plus modérées.

\section{Le typhus à tiques d'Asie}

Le typhus à tiques d'Asie, aussi connu sous le nom de rickettsiose associée à une lymphangite (LAR) est principalement observé en Sibérie et en Mongolie. Par conséquent on le connaît également sous le nom de typhus à tiques de Sibérie. Les réservoirs bactériens sont les rongeurs et les chiens. Rickettsia sibirica a été isolée pour la première fois en 1991 à partir de la tique Hyalomma asiaticum dans la région d'Alachan de la Mongolie Intérieure [18]. Depuis, cette souche a été détectée dans d'autres espèces de Hyalomma, telles que Hyalomma truncatum et Hyalomma excavatum et dans d'autres régions du monde [19]. La période d'incubation est généralement de 4 à 7 jours mais peut couvrir une période de 3 à 18 jours. La morsure de tique peut évoluer en ulcération nécrotique. Elle entraîne une rickettsiose modérée, accompagnée de légère fièvre, de céphalées, de myalgie, de lymphadénopathie locale, de symptômes du système nerveux central et d'éruptions cutanées, parfois accompagnée de purpura. Ces symptômes peuvent durer 6 à 10 jours puis disparaissent sans traitement.

\section{La fièvre boutonneuse japonaise}

La fièvre boutonneuse japonaise (ou orientale) est causée par Rickettsia japonica et est endémique dans la région du sud-ouest du Japon. Elle a été décrite pour la première fois en 1984 [20]. Il s'agit d'une fièvre boutonneuse typique [21]. Des cas d'encéphalite ont été rapportés.

\section{La fièvre pourprée méditerranéenne}

Cette maladie a été décrite pour la première fois en Tunisie en 1920 [22] et peut être observée actuellement dans les régions méditerranéennes, en Afrique Subsaharienne, en Inde et autour de la mer Noire. Elle est causée par $R$. conorii et est similaire et plus modérée que la maladie due à Rickettsia rickettsii. La tique doit rester fixée au moins $20 \mathrm{~h}$ pour qu'il y ait transmission de la maladie. Le taux de mortalité peut atteindre jusqu'à $2,5 \%$ [23].

R. conorii israelensis cause la fièvre pourprée d'Israël, et $R$. conorii caspia est à l'origine de la fièvre d'Astrakhan.

\section{Le typhus à tiques $d u$ Queensland}

Le typhus à tiques du Queensland a été identifié en 1946 au Queensland, un état de l'est de l'Australie [24]. La bactérie causant cette maladie, Rickettsia australis, est transmise par la tique Ixodes holocyclus. Le typhus à tiques du Queensland est caractérisé par une poussée de fièvre soudaine, des céphalées, une myalgie, et une éruption maculopapulaire ou vésiculaire. Ces symptômes s'accompagnent souvent d'une escarre au niveau de la morsure de tique et d'une lymphadénopathie. Une maladie similaire causée par Rickettsia honei a été observée sur l'île Flinders (en Tasmanie).

\section{La fièvre pourprée des montagnes Rocheuses}

$R$. rickettsii est observée dans la région sud du Canada, sur tout le territoire des États-Unis, au Mexique, en Amérique Centrale, au Brésil, et en Argentine. Ses réservoirs sont divers rongeurs, les lapins et les lièvres. Les vecteurs les plus importants sont Dermacentor variabilis et $D$. andersoni. Environ 500 cas de FPMR sont rapportés chaque année aux États-Unis.

La maladie [25] a été décrite pour la première fois dans les années 1890 [26] et initialement appelée la «rougeole noire». Elle se déclare brutalement 2 à 12 jours après l'exposition à la tique. Les symptômes les plus caractéristiques sont des maux de tête sévères, des myalgies et une éruption maculopapulaire typique commençant à apparaître sur les chevilles et les poignets au troisième jour. Ces symptômes s'accompagnent souvent de nausées et de vomissements, de douleurs abdominales et de conjonctivite. Non traitée, la fièvre peut durer 2 à 3 semaines et l'éruption cutanée risque de s'accompagner de pétéchies et d'ecchymoses. La mortalité chez les patients manifestant une réponse inflammatoire systémique est d'environ 20\%. Aux États-Unis, le taux de mortalité est d'environ $5 \%$. Le diagnostic sérologique (par un test d'immunofluorescence indirect) n'est pas sensible au début de l'infection. Les techniques de diagnostic fondées sur la réaction en chaîne par polymérase (de l'anglais polymerase chain reaction, PCR) ont énormément facilité le diagnostic [27]. Un diagnostic correct et un traitement adéquat débuté assez tôt peuvent sauver des vies. Le traitement de choix est la tétracycline. Il se poursuit jusqu'à ce que le patient soit apyrétique pendant 3 jours, à savoir pendant 7 à 10 jours. Lorsque la tétracycline est contre-indiquée 
(grossesse, jeunes enfants), le chloramphénicol peut être une alternative. Aucun vaccin n'existe actuellement sur le marché.

\section{La lymphadénopathie transmise par les tiques}

La maladie causée par la bactérie $R$. slovaca a été décrite pour la première fois en 1997 [28] et est transmise par la tique $D$. marginatus et probablement également par les tiques $I$. ricinus ou D. reticalus [29]. Cette lymphadénopathie se caractérise par des lésions ulcéreuses, vésiculaires ou des escarres au site de morsure de la tique puis par un grossissement douloureux des ganglions lymphatiques, accompagné de fièvre légère, d'une grande fatigue, d'un état de somnolence, de céphalées, de myalgie, d’arthralgie et d'une perte d'appétit. [30]. L'éruption cutanée est extrêmement rare [31]. R. slovaca a été observée dans de nombreux pays d'Europe. En Hongrie, la maladie est connue sous le nom de «cicatrice du bois» ou lymphadénopathie avec érythème nécrotique transmise par la tique Dermacentor [32]. Le diagnostic est établi à partir d'une biopsie du ganglion lymphatique démontrant la présence du pathogène grâce aux techniques de génétique moléculaire.

$R$. helvetica a été observée en Europe, au Japon, et en Thaïlande. En Europe 2,5 à 38\% des I. ricinus sont porteurs de $R$. helvetica $[33,34]$. La maladie causée par $R$. helvetica n'est pas encore bien définie et se manifeste par des symptômes grippaux tels que fièvre, malaises, céphalées, et myalgie, sans éruption cutanée [35].

\section{L'ehrlichiose monocytaire humaine}

L'ehrlichiose monocytaire humaine (EMH) a été décrite pour la première fois en 1987 [36].

L'Ehrlichia est une bactérie Gram-négative de 0,2 à $2 \mu \mathrm{m}$ appartenant à l'ordre des Rickettsiales. C'est un parasite intracellulaire obligatoire. Ehrlichia et Anaplasma sont des pathogènes portés par des animaux: on trouve Ehrlichia chez les chiens et Anaplasma chez les moutons, les bovins et les cerfs. L'EMH est principalement causée par la bactérie Ehrlichia chaffeensis mais d'autres espèces d'Ehrlichia ont également été identifiées comme agents responsables de la maladie. Ainsi, Ehrlichia ewingii a été identifiée chez des patients sous thérapie immunosuppressive [37] et Ehrlichia sennetsu a causé une maladie semblable à la mononucléose au Japon et en Malaisie [38]. De plus, une infection asymptomatique par Ehrlichia canis a été rapportée chez un patient au Vénézuela [39]. Aux États-Unis, l'EMH est transmise par la tique étoilée américaine (lone star tick) et probablement par d'autres espèces de tiques. De 1986 à 1997, 742 cas d'EMH ont été rapportés aux États-Unis.

Maladies émergentes transmises par les tiques
Après une période d'incubation de 12 à 14 jours, l'infection cause une maladie fébrile, avec des céphalées, une anorexie, des vomissements, et une myalgie. Ces symptômes sont souvent accompagnés de leucopénie, de thrombocytopénie et de taux d'enzymes hépatiques anormaux. Les poumons et les reins risquent d'être également endommagés et les patients peuvent être victimes d'attaque cérébrale et tomber dans le coma. Par ailleurs, une éruption maculaire ou maculopapulaire est plus fréquente chez les enfants que chez les adultes. L'EMH peut évoluer en une maladie grave à risque létal, en particulier lorsqu'elle n'est pas traitée. En raison de la nature non spécifique des symptômes, le diagnostic n'est en général pas établi. Un test PCR pour détecter l'EMH et l'anaplasmose granulocytaire humaine est disponible. La doxycycline est le traitement de choix.

\section{L'anaplasmose granulocytaire humaine}

L'anaplasmose granulocytaire humaine [40] était nommée avant 2003 l'ehrlichiose granulocytaire humaine [41].

L'Anaplasma phagocytophilum est une bactérie intracellulaire obligatoire Gram-négative de 0,5 à $2 \mu \mathrm{m}$, qui forme des microcolonies intracytoplasmiques (morulas) dans les granulocytes neutrophiles. La lyse de la cellule permet d'infecter davantage de granulocytes. Les principaux porteurs de l'anaplasmose aux États-Unis sont la tique occidentale à pattes noires et la tique du cerf, toutes deux également vecteurs de la maladie de Lyme.

En 2005, 786 cas d'anaplasmose granulocytaire humaine et 506 cas de EMH furent rapportés aux centres de contrôle des maladies et de prévention (Centers for Disease Control and Prevention, CDC). En Europe, A. phagocytophilum a été isolé pour la première fois chez un patient en Slovénie et mis en culture [42]. I. ricinus est le vecteur en Europe. Bien que A. phagocytophilum soit observé dans 14\% des genres I. ricinus en Autriche, la maladie causée par ce pathogène reste extrêmement rare. Des cas de transmission de l'A. phagocytophilum par transfusion sanguine ont été rapportés [43].

Après une période d'incubation d'une semaine (pouvant durer jusqu'à 30 jours), des symptômes grippaux se développent: fortes fièvres, frissons, malaises, céphalées, myalgie, arthralgie, vertiges, vomissements et toux sèche [44]. Ces symptômes peuvent être compliqués d'un sepsis, et d'altérations pulmonaire, cardiaque, rénale et nerveuse. La maladie peut durer de quelques jours à deux mois. Les analyses biochimiques montrent des cas de thrombocytopénie, de leucopénie, des taux d'enzymes hépatiques anormaux et une protéine $C$ réactive élevée. 
L'examen au microscope d'un frottis sanguin permet d'observer la présence typique de morulas dans les neutrophiles. Pour établir un diagnostic, un test par PCR dans un laboratoire spécialisé est plus pratique qu'une mise en culture et plus fiable qu'une détection par immunofluorescence (les anticorps pouvant persister très longtemps après l'infection). Le traitement par doxycycline pendant 7 jours permet un rétablissement très rapide. Quand la tétracycline est contre-indiquée, il est possible d'utiliser la rifampicine.

Récemment deux lignées de $A$. phagocytophilum ont été identifiées grâce à la PCR en temps réel, présentant des génotypes différents, groEL-G et groEL-A. Ce n'est qu'en 2009 que la lignée avec le génotype groEL-A a été pour la première fois observée chez l'homme, seule la lignée avec le génotype groEL-G ayant été alors identifiée chez l'homme [45].

\section{Maladies spirochétiennes transmises par les tiques}

\section{Borrelia}

La borrelia appartient à la famille des Spirochètes et possède une caractéristique unique: certaines des protéines de la membrane externe sont codées par des gènes plasmidiques. La totalité du génome a été séquencé [46]. Aux États-Unis, l'espèce B. burgdorferi sensu stricto est la plus courante alors qu'en Europe, d'autres agents pathogènes ont été identifiés: Borrelia afzelii, Borrelia garinii, Borrelia lusitaniae, Borrelia spielmani et Borrelia valaisiana. Alors que $B$. afzelii est principalement responsable de maladies cutanées (acrodermatite), B. garinii cause surtout des maladies neurologiques.

\section{La maladie de Lyme}

La maladie de Lyme a été décrite pour la première fois en 1977 chez des enfants présentant des arthrites et vivant près de Lyme, dans l'État du Connecticut aux États-Unis [47]. Elle a été identifiée comme entité en 1982 par Burgdorfer et al. [48], mais ses manifestations avaient déjà été bien décrites plusieurs années auparavant: acrodermatite chronique atrophiante (1902) [49], érythème chronique migrant (1913) [50], méningopolynévrite (1941) [51], lymphadénose cutanée bénigne (1943) [52].

Les symptômes des personnes contaminées peuvent varier énormément et affecter entre autres la peau, le système nerveux, les articulations, le cour, les ganglions lymphatiques, le foie, la rate, les yeux [53]. La majorité des patients présente une lésion cutanée bien particulière qui se développe lentement à partir du site de la morsure
Tableau 3. Symptômes cliniques d'une borréliose de Lyme

Stade 1 = infection localisée

- Érythème migrant, lymphadénopathie locale

Stade 2 = infection disséminée

- Lésions annulaires secondaires, érythème ou urticaire diffus, lymphocytome

- Douleur migratoire, arthrites brèves, myosite, ostéomyélite, panniculite

- Méningite, paralysie faciale (paralysie de Bell), encéphalite, mononévrites multiples, myélite, chorée, ataxie cérébelleuse

- Lymphadénopathie généralisée, splénomégalie

- Bloc nodal auriculo-ventriculaire, cardite

- Conjonctivite, irite, choroïdite, hémorragie rétinienne

- Hépatite modérée

- Maux de gorge

- Hématurie microscopique, protéinurie, orchite

- Malaise, fatigue

Stade 3 = infection persistante

- Acrodermatite chronique atrophiante

- Lésions sclérodermiques localisées

- Arthrite prolongée ou récidivante, enthésopathie, périostite

- Encéphalomyélite chronique, polyradiculopathie

- Kératite

(érythème migrant). Un syndrome grippal peut aussi être observé: malaise, grande fatigue, céphalées, myalgies, fièvre ou lymphadénopathie locale [54]. Sans traitement, il est possible que l'infection se dissémine et évolue vers une neuroborréliose aiguë dans 15\% des cas, avec des manifestations cliniques telles que méningite lymphocytaire avec céphalées et légère raideur de la nuque, paralysie faciale, inflammation du nerf optique, encéphalite, myélite, radiculonévrite ou ataxie cérébelleuse. Les symptômes peuvent durer des semaines, voire des mois. Après plusieurs semaines, environ 5\% des patients non traités peuvent présenter des troubles cardiaques. Des mois après l'infection, environ $60 \%$ des patients risquent de souffrir d'œè̀mes et de douleurs articulaires essentiellement au niveau du genou. Un véritable débat existe autour de l'existence, des critères, et du traitement de la maladie de Lyme chronique (tableau 3) [55].

Un diagnostic par culture de l'agent pathogène à partir de biopsies, de fluide cérébrospinal ou de sang est rarement possible. Pour diagnostiquer la maladie aiguë, la PCR n'est pas assez sensible et les autres tests (ELISA ou Western-Blot) sont souvent difficiles à interpréter. Par conséquent, il est recommandé de traiter l'érythème migrant ou le lymphocytome sans attendre les résultats d'un test, par des antibiotiques: doxycycline, amoxicilline, céfuroxime, ceftriaxone ou pénicilline, en fonction du stade 
de la maladie [56, 57]. La maladie localisée est souvent traitée par voie orale pendant 2 à 3 semaines alors que la maladie disséminée et ancienne est traitée par des antibiotiques pendant 2 à 4 semaines. Pour le traitement de l'arthrite récurrente, de la cardite ou de la neuroborréliose, une thérapie intraveineuse est préférable. Le prognostic d'une maladie de Lyme traitée est excellent.

Un vaccin recombinant contenant l'antigène de surface OspA1 de B. burgdorferi sensu stricto a été retiré du marché en 2002 après trois ans d'utilisation aux ÉtatsUnis.

La maladie de Lyme est la maladie transmise par les tiques la plus courante: entre 1992 et 2006, 248074 cas de borréliose de Lyme ont été rapportés aux CDC [58]. En Allemagne, environ 80000 cas de borréliose de Lyme (incidence supérieure à 100/100 000) et 300 à 500 cas d'ETT surviennent chaque année. En Autriche, la borréliose est la maladie transmise par les tiques la plus répandue (estimé à 4000 cas par an) suivie de l'ETT (50 à 100 cas par an). En Slovénie, où, contrairement à l'Autriche, la couverture vaccinale contre l'ETT est faible, l'ETT est endémique et représente la principale maladie transmise par les tiques. Ainsi, sur 86 enfants slovènes souffrant d'une maladie fébrile dans les 6 semaines suivant la morsure d'une tique, 33 d'entre eux avaient diverses maladies non consécutives à la morsure de tique et 28 souffraient d'une maladie associée à la morsure de tique: 18 ETT, 13 borrélioses de Lyme, 3 ehrlichioses monocytaires, 1 anaplasmose granulocytaire. De plus, 6 de ces enfants avaient plus d'un agent transmis par les tiques [59].

\section{La fièvre récurrente}

La fièvre récurrente transmise par les tiques est causée par Borrelia duttoni alors que celle transmise par les poux est causée par Borrelia recurrentis. La fièvre récurrente transmise par les tiques est observée majoritairement en Afrique, en Espagne, en Arabie Saoudite, en Asie et dans certaines régions occidentales des États-Unis et du Canada. L'agent est souvent transmis par la tique africaine molle Ornithodoros moubata.

La fièvre récurrente transmise par les tiques est une maladie multisystémique observée en Amérique du Nord, en Europe et en Asie. Après une période d'incubation de 5 à 15 jours, les patients développent les symptômes suivants: soudaine poussée de fièvre, frissons, céphalées, douleurs musculaires ou articulaires et nausées. Une éruption cutanée peut également apparaître. Ces symptômes peuvent durer 2 à 9 jours et se répéter pendant plusieurs semaines si le patient n'est pas traité. Le traitement consiste en une antibiothérapie de 1 à 2 semaines avec de la tétracycline et le rétablissement est rapide. Ce traitement peut entraîner une réaction de Jarisch-Herxheimer, probablement induite par le TNF- $\alpha$ (facteur de nécrose tumorale), accompagnée de diaphorèse, fièvre, tachypnée, tachycardie et hypotension. La fièvre récurrente n'entraîne que rarement des complications graves et la mort.

\section{Autres maladies bactériennes rares transmises par les tiques}

\section{La fièvre $Q$}

La fièvre Q est causée par la bactérie Coxiella burnetii, un coque aérobie à Gram négatif de $0,4 \mu \mathrm{m}$ et appartenant à la famille des Coxiellaceae, de l'ordre des Legionellales. C'est une bactérie intracellulaire, décrite pour la première fois en Australie en 1937 [60]. Cet organisme est peu répandu. Ses réservoirs naturels sont les bovins, les moutons, les chèvres, et d'autres mammifères domestiques, tels que les chats et les chiens. L'infection provient de l'inhalation de particules aéroportées infectées ou du contact avec le lait, l'urine, les selles, les sécrétions vaginales ou le sperme d'animaux infectés. Cette bactérie est extrêmement durable dans l'environnement. On peut la considérer comme la maladie la plus infectieuse au monde car une seule bactérie est suffisante pour contaminer un homme. La transmission par les tiques est possible mais rare. La transmission d'homme à homme a été rapportée dans un nombre très limité de cas.

La période d'incubation varie de 9 à 40 jours mais est habituellement de 2 à 3 semaines. La maladie se manifeste le plus souvent par des symptômes grippaux: poussée de fièvre brutale, malaises, sueurs abondantes, céphalées sévères, myalgie, douleurs articulaires, perte d'appétit, troubles des voies respiratoires supérieures, toux sèche, douleur pleurétique, frissons, confusion et symptômes gastro-intestinaux tels que nausées, vomissements et diarrhées. La fièvre dure approximativement 7 à 14 jours. La maladie peut évoluer progressivement vers une pneumonie atypique, pouvant entraîner un syndrome de détresse respiratoire aigüe à risque mortel. Moins souvent, la fièvre Q peut induire une hépatite granulomateuse avec malaises, fièvre, douleur abdominale et jaunisse. L'endocardite [61] est une complication tardive de la fièvre Q, pouvant survenir des mois ou des années après l'infection, et pouvant avoir un taux de mortalité d'environ $10 \%$, même chez les patients traités.

Le traitement de la fièvre $Q$ consiste en une antibiothérapie à base de doxycycline, en cas de grossesse, 5 semaines de co-trimoxazole. 
Un vaccin (intradermique) à base de germes atténués entiers est disponible en Australie (une dose unique est suffisante). Des examens sanguins et cutanés sont nécessaires avant la vaccination pour identifier une éventuelle immunité préexistante, les personnes déjà immunisées risquant de développer des réactions locales graves.

\section{La tularémie}

La tularémie est également connue sous le nom de fièvre du lapin. En 1922, Francisella tularensis a été reconnue comme l'agent responsable de la tularémie [62]. Francisella est un genre de bactéries pathogènes à Gram négatif en forme de bâtonnet ou coccoïde, aérobie facultative. Ce sont des parasites intracellulaires des macrophages. Il en existe deux types: A et $\mathrm{B}$. Les réservoirs principaux sont les rongeurs (campagnols, rats musqués, et castors), les eaux contaminées et la boue. La bactérie Francisella peut survivre plusieurs semaines en milieu naturel. Le lapin domestique est la première source d'infection chez l'homme. La transmission se fait par contact direct, par des bactéries aérosolisées, par l'ingestion de tissue infecté ou par la morsure de tique ou de puce infectées. Les lapins sauvages n'étant plus vendus sur les marchés, la tularémie est devenue une maladie rare.

La forme clinique de la tularémie reflète son mode de transmission. On en distingue trois: la forme typhoïde avec une prédominance de symptômes systémiques, la forme pulmonaire et la forme ulcéroglandulaire avec symptômes locaux (80\%). Après inoculation par morsure de tique (11\%) [63], une papule ou un ulcère peut se développer, suivi d'un gonflement des ganglions lymphatiques et parfois de fièvre. Une enquête récente réalisée au Missouri (190 cas observés de 2000 à 2007) a révélé que les enfants présentent préférentiellement une tularémie glandulaire alors que le diagnostic de tularémie pulmonaire concerne plutôt les adultes. De plus, parmi les 78 cas ayant une source d'exposition connue, $72 \%$ d'entre eux étaient associés à une morsure de tique [64]. Sans traitement, la lymphadénopathie risque de durer des mois. Le taux de mortalité de toutes les formes cliniques confon- dues est d'environ $8 \%$ sans traitement et $1 \%$ avec antibiothérapie.

Lors d'un diagnostic par culture, il est important d'informer le laboratoire de la présence éventuelle de F. tularensis dans l'échantillon pour que d'une part un milieu spécifique soit utilisé et que d'autre part, les précautions nécessaires soient prises de façon à éviter une contamination du laboratoire. Le traitement de choix consiste en une antibiothérapie par streptomycine, bien que la tétracycline, les aminoglycosides ou les fluoroquinolones aient déjà été utilisés.

\section{Maladies parasitaires dues à des protozoaires et transmises par les tiques}

En Europe, les (rares) infections humaines sont souvent causées par Babesia divergens, alors qu'aux ÉtatsUnis on trouve plus communément Babesia microti. La babésiose [65] a été identifiée pour la première fois dans le nord est des États-Unis dans les années 60. Les hôtes naturels sont la souris à pattes blanches, et les campagnols des champs. Une morsure de tiques Ixodes dammini est en général la source de l'infection humaine. Plus la tique demeure longtemps fixée à la peau, plus le risque de transmission de sporozoïtes est élevé. L’infection peut également être transmise par transfusion sanguine.

Les parasites envahissent les globules rouges et se répliquent à l'intérieur. En raison de leur apparence en forme de poire à l'intérieur des cellules infectées, ils sont appelés «piroplasmes». Ils peuvent être responsables d'hémolyse avec toutes les conséquences que cela entraîne. La mortalité peut s'avérer très élevée [66]. Chez l'homme, l'infection peut être subclinique ou se manifester dans les 10 à 24 jours suivant la morsure de tique par des symptômes fébriles et une anémie. Les manifestations sont plus sévères chez les personnes agées, les patients immunodéprimés ou les sujets aspléniques [67]. Le traitement consiste en l'administration d'azithromycine et d'atovaquone.

\section{Bibliographie}

1 Drutz JE: Arthropods; in Feigin RD, Cherry JD, Demmler GJ, Kaplan SL (eds): Textbook of Pediatric Infectious Diseases, ed 5. Philadelphia, Saunders, 2004, pp 28352836.

2 Goodman JL, Dennis DT, Sonenshine DE (eds): Tick-Borne Diseases of Humans. Washington, ASM Press, 2005.
3 Gothe R, Kunze K, Hoogstraal H: The mechanisms of pathogenicity in the tick paralyses. J Med Entomol 1979;16:357-369.

$\checkmark 4$ Casals J: Antigenic similarity between the virus causing Crimean hemorrhagic fever and Congo virus. Proc Soc Exp Biol Med 1969; 131:233-236. 
$\checkmark 5$ Burt FJ, Swanepoel R, Shieh WJ, et al: Immunohistochemical and in situ localization of Crimean-Congo hemorrhagic fever (CCHF) virus in human tissues and implications for CCHF pathogenesis. Arch Pathol Lab Med 1997;121:839-846.

6 Burt FJ, Swanepol R: Crimean-Congo hemorrhagic fever; in Goodman JL, Dennis DT, Sonenshine DE (eds): Tick-Borne Diseases of Humans. Washington, ASM Press, 2005, pp 164-175.

7 Tavakoli NP, Wang H, Dupuis M, et al: Fatal case of deer tick virus encephalitis. N Engl J Med 2009;360:2099-2107.

8 Pattnaik P: Kyasanur forest disease: an epidemiological view in India. Rev Med Virol 2006;16:151-165.

-9 Adhikari Prabha MR, Prabhu MG, Raghuveer $C V$, et al: Clinical study of 100 cases of Kyasanur forest disease with clinicopathological correlation. Indian J Med Sci 1993;47: 124-130.

10 Lin D, Li L, Dick D, et al: Analysis of the complete genome of the tick-borne flavivirus Omsk hemorrhagic fever virus. Virology 2003;313:81-90.

11 Schneider H: Über epidemische akute 'Meningitis serosa'. Wien Klin Wochenschr 1931; 44:350-352.

12 Zoehrer B, Spork D, Zenz W: Frühsommermeningoenzephalitis - Klinik und Therapie. Monatsschr Kinderheilkd 2003;151:11631169.

13 Mutz I, Kunz C, Holzmann H: Prävention der Frühsommermeningoenzephalitis. Monatsschr Kinderheilkd 2003;151:11701177.

14 Kunz C: TBE vaccination and the Austrian experience. Vaccine 2003;21:S1/50-S1/55.

15 Chapman AS, Bakken JS, Folk SM, et al; Tickborne Rickettsial Diseases Working Group: Diagnosis and management of tickborne rickettsial diseases: Rocky Mountain spotted fever, ehrlichiosis, and anaplasmosis - United States: a practical guide for physicians and other health-care and public health professionals. MMWR Recomm Rep 2006;55(RR-4):1-27.

16 Bogdan C: Rickettsiaceae (Rickettsia, Orientia), Anaplasmataceae (Anaplasma, Ehrlichia, Neorickettsia) and Coxiellaceae; in Hahn H, Kaufmann SHE, Thomas F, Suerbaum S (eds): Medizinische Mikrobiologie und Infektiologie, ed 6. Berlin, Springer, 2009.

$\checkmark 17$ Dumler JS, Barbet AF, Bekker CPJ, et al: Reorganization of genera in the families Rickettsiaceae and Anaplasmataceae in the order Rickettsiales: unification of some species of Ehrlichia with Anaplasma, Cowdria with Ehrlichia, and Ehrlichia with Neorickettsia, description of six new species combinations and designation of Ehrlichia equi and 'HGE agent' as subjective synonyms of Ehrlichia phagocytophilum. Int J Syst Evol Microbiol 2001;51:2145-2165.
18 Yu X, Fan M, Xu G, et al: Genotypic and antigenic identification of two new strains of spotted fever group rickettsiae isolated from China. J Clin Microbiol 1993;31:83-88.

19 Psaroulaki A, Germanakis A, Gikas A, et al: Simultaneous detection of 'Rickettsia mongolotimonae' in a patient and a tick in Greece. J Clin Microbiol 2005;43:3558-3559.

20 Mahara F: Japanese spotted fever: report of 31 cases and review of the literature. Emerg Infect Dis 1997;3:105-111.

21 Mahara F: Japanese spotted fever: report of 31 cases and review of the literature. Emerg Infect Dis 1997;3:105-111.

22 Conor A, Bruch A: Une fièvre éruptive observée en Tunisie. Bull Soc Pathol Exot Filial 1910;8:492-496.

23 Raoult D, Weiller PJ, Chagnon A, et al: Mediterranean spotted fever: clinical, laboratory, and epidemiological features of 199 cases. Am J Trop Med Hyg 1986;35:845-850.

24 Plotz H, Smadel JE, Bennet BI: North Queensland tick typhus: studies of the aetiological agent and its relation to other rickettsial diseases. Med J Aust 1946;2:263-268.

25 Walker DH, Lane TW: Rocky Mountain spotted fever: clinical signs, symptoms, and pathophysiology; in Walker DH (ed): Biology of Rickettsial Diseases. Boca Raton, CRC Press, 1988, vol 1, pp 63-78.

26 Philip RN: Rocky Mountain Spotted Fever in Western Montana: Anatomy of a Pestilence. Hamilton, Bitter Root Valley Historical Society, 2000.

27 Regnery RL, Spruill CL, Plikatys BD: Genotypic identification of rickettsiae and estimation of intraspecies sequence divergence for portions of two reckettsial genes. J Bacteriol 1991;173:1576-1589.

28 Raoult D, Berbis P, Roux V, et al: A new ticktransmitted disease due to Rickettsia slovaca. Lancet 1997;350:112-113.

29 Rehacek J, Kocianova E, Lukacova M, et al: Detection of spotted fevergroup (SFG) rickettsia in Ixodes ricinus ticks in Austria. Acta Virol 1997;41:355-356.

30 Lakos A: Tick-borne lymphadenopathy (TIBOLA). Wien Klin Wochenschr 2002;114: 647-653.

31 Raoult D, Lakos A, Fenollar F, et al: Spotless rickettsiosis caused by Rickettsia slovaca and associated with Dermacentor ticks. Clin Infect Dis 2002;34:1331-1336.

32 Oteo JA, Ibarra V: DEBONEL (Dermacentor-borne necrosis erythema lymphadenopathy). A new tick-borne disease? Enferm Infect Microbiol Clin 2002;20:51-52.

33 Fournier PE, Grunnenberger F, Jaulhac B, et al: Evidence of infection in humans with Rickettsia helvetica in Eastern France. Emerg Infect Dis 2000;6:389-392.

34 Nillson K, Lindquist O, Liu AJ, et al: Rickettsia helvetica in Ixodes ricinus ticks in Sweden. J Clin Microbiol 1999;37:400-403.
35 Fournier PE, Allombert C, Supputamongkol $\mathrm{Y}$, et al: Aneruptive fever associated with antibodies to Rickettsia helvetica in Europe and Thailand. J Clin Microbiol 2004;42:816-818.

36 Maeda K, Markowitz N, Hawley RC, et al: Human infection with Ehrlichia canis, a leukocytic rickettsia. N Engl J Med 1987;316: 853-856.

37 Buller RS, Arens M, Hmiel SP, et al: Ehrlichia ewingii, a newly recognized agent of human ehrlichiosis. N Engl J Med 1999;341: 148-155.

38 Misao T, Kobayashi Y: Studies on infectious mononucleosis: isolation of an etiologic agent from blood, bone marrow, and lymph node of a patient with infectious mononucleosis by using mice. Tokyo Iji Shinshi 1954;71: 683-686.

- 39 Perez M, Rikihisa Y, Wen B: Ehrlichia canislike agent isolated from a man in Venezuela: antigenic and genetic characterization. J Clin Microbiol 1996;34:2133-2139.

-40 Dumler JS, Choi K, Garcia-Garcia JC, et al: Human granulocytic anaplasmosis and Anaplasma phagocytophilum. Emerg Infect Dis 2005; 11:1828-1834.

41 Walder G, Falkensammer B, Aignes J, et al: First documented case of human granulocytic ehrlichiosis in Austria. Wien Klin Wochenschr 2003;115:263-266.

42 Petrovec M, Lotric Furlan S, Zupanc TA, et al: Human disease in Europe caused by a granulocytic Ehrlichia species. J Clin Microbiol 1997;35:1556-1559.

43 Centers for Disease Control: Anaplasma phagocytophilum transmitted trough blood transfusion - Minnesota, 2007. MMWR Morb Mortal Wkly Rep 2008;57:1145-1148.

44 Lotric-Furlan S, Rojko T, Petrovec M, et al: Epidemiological, clinical, and laboratory characteristics of patients with human granulocytic anaplasmosis in Slovenia. Wien Klin Wochenschr 2006;118:708-713.

45 Haschke-Becher E, Bernauer R, Walleczek AM, et al: First detection of the Anaplasma phagocytophilum groEL-A genotype in man. J Infect, submitted.

46 Fraser CM, Casjens S, Huang WM, et al: Genomic sequence of a Lyme spirochete, Borrelia burgdorferi. Nature 1997;390:580-586.

47 Steere AC, Malwista SE, Snydman DR, et al: Lyme arthritis: an epidemic of oligoarticular arthritis in children and adults in three Connecticut communities. Arthritis Rheum 1977;20:7-17.

48 Burgdorfer W, Barbour AG, Hayes SF, et al: Lyme disease - a tick-borne spirochetosis? Science 1982;216:1317-1319.

49 Herxheimer K, Hartman K: Über Acrodermatitis chronica atrophicans. Arch Dermatol Syph 1902;61:57-76.

50 Lipschütz B: Über eine seltene Erythemform (Erythema chronicum migrans). Arch Dermatol Syph 1913;118:349-356. 
-51 Bannwarth A: Chronische lymphozytäre Meningitis, entzündliche Polyneuritis und 'Rheumatismus'. Arch Psychiatr Nervenkrankh 1941;113:284-376.

52 Bäfverstedt B: Über Lymphadenosis benigna cutis. Acta Derm Venereol (Stockh) 1943; 24(suppl 11):1-202.

53 Stanek G, Strle F: Lyme borreliosis. Lancet 2003;362:1639-1647.

54 Steere AC: Lyme disease. N Engl J Med 2001; 345:115-125.

-55 Feder HM, Johnson BJB, O'Connell S, et al: A critical appraisal of 'chronic Lyme disease'. N Engl J Med 2007;357:1422-1430.

- 56 Wormser GP, Nadelman RB, Dattwyler RJ, et al: Practice guidelines for the treatment of Lyme disease. Clin Infect Dis 2000;31(suppl 1):S1-S14
57 Stanek G: Borreliose update. Antibiotika Monitor 2007;XXIII:1/07.

58 Centers for Disease Control: Surveillance for Lyme disease - United States, 1992-2006. MMWR Morb Mortal Wkly Rep 2008;57: 1-9.

59 Arnež M, Lužnik-Bufon T, Avšic-Zupanc T, et al: Causes of febrile illness after a tick bite in Slovenian children in 2001 within 6 weeks after tick bite. Pediatr Infect Dis J 2003;22: 1078-1083.

60 Derrick EH: Q fever, a new fever entity: clinical features, diagnosis and laboratory investigation. Med J Aust 1937;11:281-299.

61 Karakousis PC, Trucksis M, Dumler JS: Chronic Q fever in the United States. J Clin Microbiol 2006;44:2283-2287.

62 Hornick R: Tularemia revisited. N Engl J Med 2001;345:1637-1639.
63 Gurycova D, Vyrostekova V, Khanakah G, et al: Importance of surveillance of tularaemia natural foci in the known epidemic area of Central Europe, 1991-1997. Wien Klin Wochenschr 2001;113:433-438.

64 Tularemia - Missouri, 2000-2007. MMWR Morb Mortal Wkly Rep 2009;58:744-748.

65 Kjemtrup AM, Conrad PA: Human babesiosis: an emerging tick-borne disease. Int J Parasitol 2000;30:1323-1337.

66 Gorenflot A, Moubri K, Precigout E, et al: Human babesiosis. Ann Trop Med Parasitol 1998;92:489-501.

67 Centers for Disease Control: Epidemiological notes and reports. Babesiosis - Connecticut. MMWR Morb Mortal Wkly Rep 1989; 38:649-650. 
